\title{
New Dosimetric Interpretation of the DV50 Vessel-Steel Experiment Irradiated in the OSIRIS MTR Reactor Using the Monte-Carlo Code TRIPOLI-4 ${ }^{\circledR}$
}

\author{
Fadhel Malouch ${ }^{\mathrm{a}}$ \\ Alternative Energies and Atomic Energy Commission (CEA), Saclay Center, \\ DEN/DANS/DM2S/SERMA, 91191 Gif-sur-Yvette Cedex, France
}

\begin{abstract}
An irradiation program DV50 was carried out from 2002 to 2006 in the OSIRIS material testing reactor (CEA-Saclay center) to assess the pressure vessel steel toughness curve for a fast neutron fluence ( $\mathrm{E}>1 \mathrm{MeV}$ ) equivalent to a French 900-MWe PWR lifetime of 50 years. This program allowed the irradiation of 120 specimens out of vessel steel, subdivided in two successive irradiations DV50 $\mathrm{n}^{\circ} 1$ and DV50 $\mathrm{n}^{\circ} 2$. To measure the fast neutron fluence $(\mathrm{E}>1 \mathrm{MeV})$ received by specimens after each irradiation, sample holders were equipped with activation foils that were withdrawn at the end of irradiation for activity counting and processing. The fast effective cross-sections used in the dosimeter processing were determined with a specific calculation scheme based on the Monte-Carlo code TRIPOLI-3 (and the nuclear data ENDF/B-VI and IRDF-90). In order to put vesselsteel experiments at the same standard, a new dosimetric interpretation of the DV50 experiment has been performed by using the Monte-Carlo code TRIPOLI- 4 and more recent nuclear data (JEFF3.1.1 and IRDF-2002). This paper presents a comparison of previous and recent calculations performed for the DV50 vessel-steel experiment to assess the impact on the dosimetric interpretation.
\end{abstract}

\section{Introduction}

Within the framework of the French NPPs lifetime extension program, an R\&D irradiation program DV50 was carried out from 2002 to 2006 in the OSIRIS material testing reactor (CEA-Saclay center) [1] to assess the pressure vessel steel toughness curve for a fast neutron fluence $(E>1 \mathrm{MeV})$ equivalent to a 900-MWe PWR lifetime of 50 years.

Material Toughness (resistance to crack growth) is governed by the energy absorbed as the crack moves forward. This mechanical property depends on chemical composition, temperature and also neutron fluence for a material under irradiation. The nil-ductility temperature (above which a material is ductile and below which it is brittle) increases with the fluence of fast neutrons $(\mathrm{E}>1 \mathrm{MeV})$.

The DV50 program allowed the irradiation of 120 CHARPY specimens out of 16MND5 vessel steel, subdivided in two successive irradiations DV50 $\mathrm{n}^{\circ} 1$ and DV50 $\mathrm{n}^{\circ}$. To measure the fast neutron fluence

\footnotetext{
${ }^{\text {a }}$ Corresponding author: fadhel.malouch@cea.fr
}

This is an Open Access article distributed under the terms of the Creative Commons Attribution License 2.0, which permits unrestricted use, distribution, and reproduction in any medium, provided the original work is properly cited. 
$(\mathrm{E}>1 \mathrm{MeV})$ received by specimens after each irradiation, sample holders were equipped with activation foils that were withdrawn at the end of irradiation for activity counting and processing. The dosimeter processing (giving neutron fluences and fluxes from dosimeter activities) needs fast effective crosssections (ratio of reaction rate and fast flux) determined with a specific calculation scheme simulating the experiment irradiation in the OSIRIS reactor.

The dosimetric interpretation initially performed for the DV50 experiment was based on the following tools and nuclear data:

- The Monte-Carlo code TRIPOLI-3 [2],

- The nuclear data evaluation ENDF/B-VI [3],

- The dosimetric response library IRDF-90 [4].

In order to put vessel-steel experiments at the same standard, a new dosimetric interpretation of the DV50 experiment has been performed by using more recent tools and nuclear data:

- The Monte-Carlo code TRIPOLI-4 [5],

- The nuclear data evaluation JEFF3.1.1 [6],

- The dosimetric response library IRDF-2002 [7].

TRIPOLI-4 code has been validated against experimental results based on neutron flux measurements performed in ex-core and in-core experiments in the case of the OSIRIS reactor [8, 9]. This paper presents a comparison of previous and recent calculations performed for the DV50 vessel steel experiment to assess the impact on the dosimetric interpretation.

In this paper, DV50 experiment and its surveillance dosimetry will first be described. Then, the initial dosimetric interpretation of the DV50 experiment will be presented. Afterwards, the methodology and results of the new dosimetric interpretation will be detailed. Finally, results of initial and new interpretations will be compared and discussed.

\section{DV50 Experiment}

\subsection{OSIRIS Reactor and DV50 Irradiation Position}

OSIRIS is a material testing reactor located at the CEA-Saclay site and operated since 1966. It is a 70 MWth pool type light water reactor with an open core. The core is a compact unit $(70 \times 80 \times$ $\left.90 \mathrm{~cm}^{3}\right)$. The core tank contains a centrally located rack containing 56 cells $\left(8 \times 8 \times 90 \mathrm{~cm}^{3}\right.$, see Fig. 1). These cells are loaded with 38 standard fuel elements, 6 control elements (hafnium absorber in upper part and fuel in lower part) and up to 7 beryllium elements (in row 10, south side). The remaining cells (in particular 24, 44 and 64) are dedicated to in-core experiments with high fast neutron flux (about $2 \times 10^{14} \mathrm{n} \cdot \mathrm{cm}^{-2} \cdot \mathrm{s}^{-1}$ ).

External grids surround the core, on 3 faces of the tank (north, east and west), making it possible to set up to 27 experimental devices on the first periphery (where the fast neutron flux is about 10 times less important than in the core) and many others on the second and the third peripheries.

The DV50 irradiation was carried out in the position L10 of the second periphery north and behind a stainless-steel block located at the position L9 of the first periphery for gamma shielding.

\subsection{DV50 Device}

The DV50 program allowed the irradiation of 120 CHARPY specimens out of 16MND5 vessel steel, subdivided in two successive irradiations DV50 n 1 (261.26 EFPD from May 2002 to November 2003) and DV50 n 2 (252.45 EFPD from January 2004 to September 2005). For each irradiation, 60 specimens were piled-up inside the IRMA5 irradiation device and irradiated in the OSIRIS ex-core position L10 


\section{$15^{\text {th }}$ ISRD}

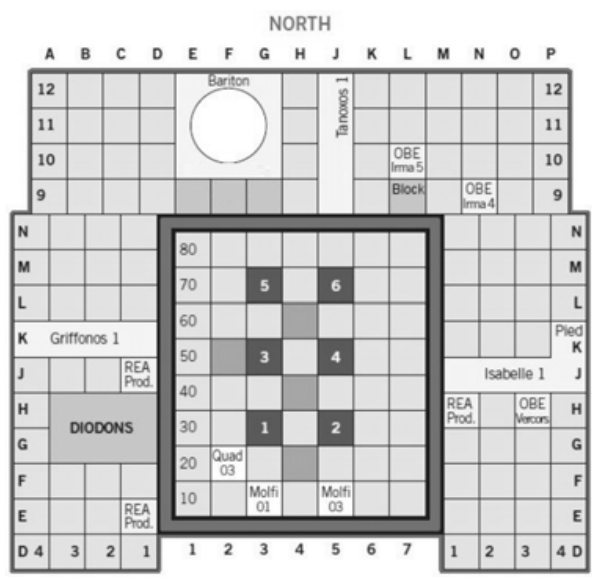

Figure 1. Schematic representation of the core of the OSIRIS reactor in its tank, the six control rods, and the incore and ex-core experimental positions. The DV50 irradiation was carried out in the ex-core position L10 of the second periphery north (behind a stainless-steel block located at the position L9 of the first periphery).
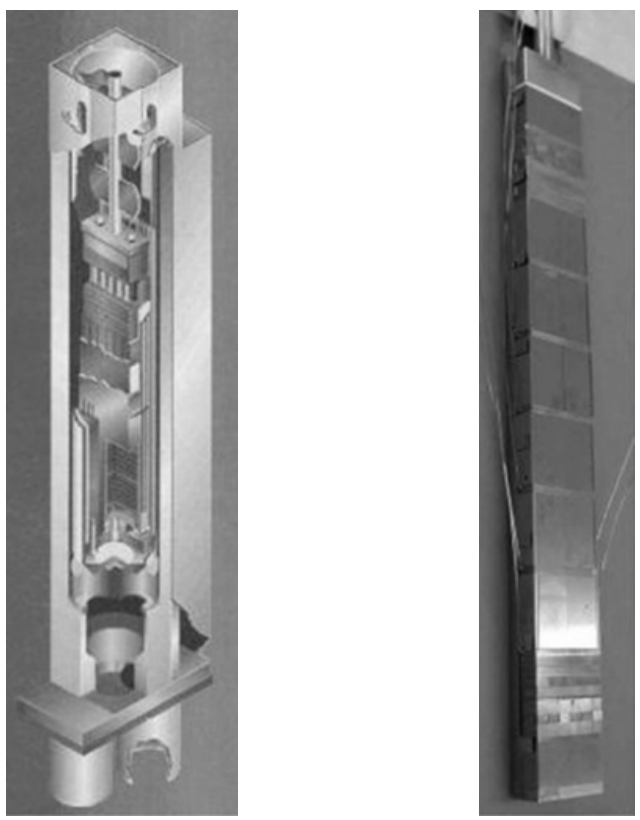

Figure 2. On the left, the IRMA capsule equipped with heating elements. On the right, 60 CHARPY specimens out of 16MND5 vessel steel steel piled-up in the DV50 sample-holder.

of the second periphery north. IRMA is a cylindrical capsule ( $75 \mathrm{~mm}$ in external diameter) designed for the irradiation, in an inert environment, of structural materials and more particularly, the steels used in pressurized water reactors. It comprises a double jacket (constituting a heat shield) equipped with heating elements distributed along the capsule to regulate the temperature of irradiated specimens (Fig. 2). 


\subsection{DV50 Dosimetry}

To measure the neutron fluence received by specimens after each irradiation DV50 $\mathrm{n}^{\circ} 1$ and DV50 $\mathrm{n}^{\circ} 2$, sample holders were equipped with activation foils that were withdrawn at the end of irradiation for activity counting and processing. Different types of dosimeters were used: nickel $(\mathrm{Ni})$, copper $(\mathrm{Cu})$, iron $(\mathrm{Fe})$, niobium $(\mathrm{Nb})$ foils for fast fluence; copper-cobalt $(\mathrm{CuCo})$ foils for thermal fluence. Fast dosimeters were placed along the sample holder at 7 axial levels; each level contains a set of 4 foils: $\mathrm{Ni}, \mathrm{Cu}, \mathrm{Fe}$ and $\mathrm{Nb}$. Thus, each type of dosimeters can provide an axial distribution of fast fluence. Using different types of foils allows us to investigate consistent results among them in terms of axial neutron flux profiles and levels.

\section{Initial Dosimetric Interpretation of the DV50 Experiment}

At the end of each irradiation DV50 $\mathrm{n}^{\circ} 1$ and DV50 $\mathrm{n}^{\circ}$, activation foils ( $\mathrm{Ni}, \mathrm{Cu}, \mathrm{Fe}$ and $\mathrm{Nb}$ ) equipping the sample holder were withdrawn for activity counting and processing.

A given dosimeter under neutron flux $\phi(E, t)$ for an irradiation time $T$ becomes more or less radioactive according to a neutron reaction of interest characterized by a microscopic cross-section $\sigma(E)$. Using an appropriate post-irradiation dosimeter processing, we can deduce from the dosimeter activity measured after irradiation an experimental "microscopic reaction rate (integrated in time)" $\tau$, which can be directly compared to the one obtained by neutron calculation (as the convolution of the cross-section and the flux distribution):

$$
\tau=\int_{0 \mathrm{MeV}}^{\infty} \int_{T} \sigma(E) \phi(E, t) d E d t
$$

The post-irradiation dosimeter processing consists essentially of the correction of the measured activity due to the foil depletion under neutron flux and the foil decay during and after irradiation. The variation of the flux amplitude (due to the reactor power variation) is also taken into account. Thanks to these corrections, the final experimental microscopic reaction rate can be directly compared to the microscopic reaction rate obtained by neutron calculation (normalized at the reactor rated power).

Fast neutron fluence $(\mathrm{E}>1 \mathrm{MeV}) \Phi_{E>1 \mathrm{MeV}}$ can be experimentally determined from the experimental microscopic reaction rate (integrated in time) $\tau$ of a given dosimeter, using a calculated "fast effective cross-section" $\sigma_{E>1 \mathrm{MeV}}$ :

$$
\Phi_{E>1 \mathrm{MeV}}=\frac{\tau}{\sigma_{E>1 \mathrm{MeV}}} .
$$

At the end of the dosimeter processing, 4 axial distributions of fast neutron fluence were obtained corresponding to the 4 types of fast dosimeters $(\mathrm{Fe}, \mathrm{Ni}, \mathrm{Nb}$ and $\mathrm{Cu}$ ). The final measured distribution of fast fluence was the average of the four ones.

For the neutron calculation of the fast effective cross-section, we assume that the neutron energy spectrum does not vary or varies little during the irradiation:

$$
\phi(E, t)=\varphi(E) .
$$

Therefore, the fast effective cross-section for a dosimeter would be the ratio of the calculated reaction rate and fast flux:

$$
\sigma_{E>1 \mathrm{MeV}}=\frac{\tau}{\Phi_{E>1 \mathrm{MeV}}}=\frac{\int_{0 \mathrm{MeV}}^{\infty} \sigma(E) \varphi(E) d E}{\int_{1 \mathrm{MeV}}^{\infty} \varphi(E) d E} .
$$




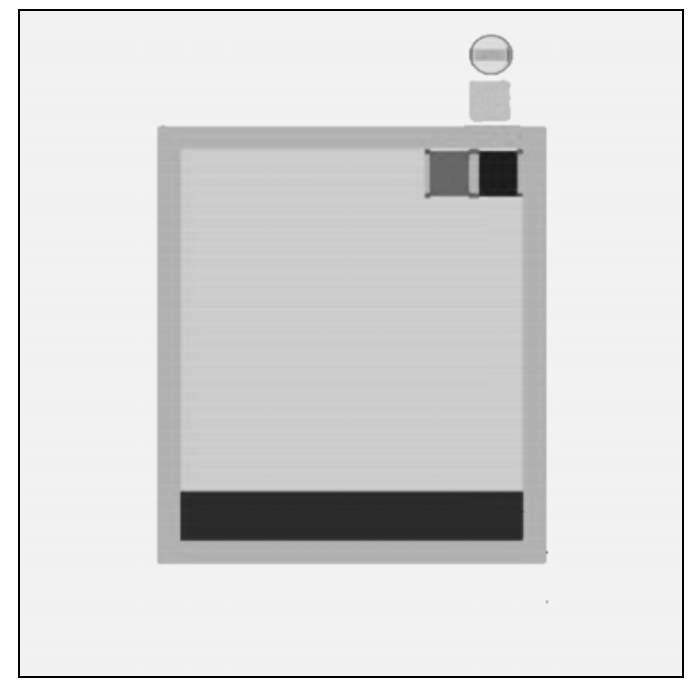

Figure 3. Geometry modeling of the OSIRIS core and the DV50 experiment with the TRIPOLI-3 code. The core geometry was performed with homogenous blocks of fuel elements.

Fast effective cross-sections used in the initial dosimetric interpretation of DV50 experiment were determined with a specific calculation scheme based on:

- The Monte-Carlo code TRIPOLI-3 used for simulating the DV50 experiment in the OSIRIS reactor,

- Its associated multigroup-wise data library from the nuclear data evaluation ENDF/B-VI for Monte-Carlo transport simulation,

- The dosimetric response library IRDF-90 used for the fast dosimeters $\mathrm{Ni}, \mathrm{Cu}, \mathrm{Fe}$ and $\mathrm{Nb}$.

In the TRIPOLI-3 calculation, a 3D-modeling of the core geometry was performed with homogenous blocks of fuel elements (Fig. 3). This was a fixed-source simulation using a neutron-source 2Ddistribution based on a power map calculated by the 2D-diffusion code DAIXY [10] (used in the operation of the OSIRIS reactor); a cosine-shaped profile was considered for the axial distribution and taken from previous measurements in the OSIRIS core. The neutron-source intensity was normalized to correspond to the rated power of the OSIRIS reactor (70 MWth).

DV50 devices ( $\mathrm{n}^{\circ} 1$ and $\mathrm{n}^{\circ}$ ) were identical, so one TRIPOLI-3 calculation was performed for both. The fast effective cross-sections should be the same for these two devices. Nevertheless, some correction factors (called "unbiaising factors" issued from different feedbacks and benchmarks) were used to correct only the fast effective cross-sections of DV $50 \mathrm{n}^{\circ} 1$, but not those of DV $50 \mathrm{n}^{\circ} 2$ (the reason was not clearly stated in the DV50-dosimetry reports emitted in 2005-2006). These corrections are less than $3 \%$ for $\mathrm{Fe}, \mathrm{Ni}$ and $\mathrm{Nb}$ dosimeters and about $10 \%$ for $\mathrm{Cu}$ dosimeters. Finally, different values of the fast effective cross-sections were used for DV50 $\mathrm{n}^{\circ} 1$ and DV50 $\mathrm{n}^{\circ} 2$ irradiations in the initial dosimetric interpretation of DV50 experiment (see Table 1).

\section{New Dosimetric Interpretation of the DV50 Experiment}

The principle of this new dosimetric interpretation of the DV50 experiment is to reassess the fast effective cross-sections used in the dosimeter processing by using more recent tools and nuclear data, in 
Table 1. Fast effective cross-sections used in the initial dosimetric interpretation of DV50 experiment. Values were issued form TRIPOLI-3 calculation (standard deviation is about $4 \%$ for $\mathrm{Fe}, \mathrm{Ni}$ and $\mathrm{Nb}$ dosimeters, and about $6 \%$ for $\mathrm{Cu}$ dosimeters) and multiplied by correction factors only in the case of the DV50 $\mathrm{n}^{\circ} 1$ irradiation.

\begin{tabular}{|c|c|c|c|c|c|}
\hline \multicolumn{2}{|c|}{ Dosimeter } & Iron & Nickel & Niobium & Copper \\
\hline \multicolumn{2}{|c|}{ Reaction of interest } & ${ }^{54} \mathrm{Fe}(\mathrm{n}, \mathrm{p})^{54} \mathrm{Mn}$ & ${ }^{58} \mathrm{Ni}(\mathrm{n}, \mathrm{p})^{58} \mathrm{Co}$ & ${ }^{93} \mathrm{Nb}\left(\mathrm{n}, \mathrm{n}^{\prime}\right)^{93 m} \mathrm{Nb}$ & ${ }^{63} \mathrm{Cu}(\mathrm{n}, \alpha)^{60} \mathrm{Co}$ \\
\hline Fast effective cross- & DV50 $\mathrm{n}^{\circ} 1$ & 83.28 & 116.57 & 200.97 & 0.6685 \\
section (mbarn) & DV50 n $^{\circ} 2$ & 85.24 & 115.53 & 197.03 & 0.6061 \\
\hline
\end{tabular}

order to put vessel-steel experiments at the same standard. Thus, a new calculation has been performed based on:

- The Monte-Carlo code TRIPOLI-4 used for simulating the DV50 experiment in the OSIRIS reactor,

- Its associated point-wise data library from the nuclear data evaluation JEFF3.1.1 for Monte-Carlo transport simulation,

- The dosimetric response library IRDF-2002 used for the fast dosimeters $\mathrm{Ni}, \mathrm{Cu}, \mathrm{Fe}$ and $\mathrm{Nb}$.

For a given dosimeter $i$, the microscopic reaction rate (integrated in time) $\tau$ is the amount directly accessible by neutron measurement and therefore independent from the fast effective cross-section used in the dosimeter processing. So, we have:

$$
\begin{gathered}
\tau=\left(\sigma_{E>1 \mathrm{MeV}}^{i}\right)^{T 3} \cdot\left(\Phi_{E>1 \mathrm{MeV}}^{i}\right)^{T 3}=\left(\sigma_{E>1 \mathrm{MeV}}^{i}\right)^{T 4} \cdot\left(\Phi_{E>1 \mathrm{MeV}}^{i}\right)^{T 4} \\
\left(\Phi_{E>1 \mathrm{MeV}}^{i}\right)^{T 4}=\frac{\left(\sigma_{E>1 \mathrm{MeV}}^{i}\right)^{T 3}}{\left(\sigma_{E>1 \mathrm{MeV}}^{i}\right)^{T 4}} \cdot\left(\Phi_{E>1 \mathrm{MeV}}^{i}\right)^{T 3}
\end{gathered}
$$

With:

- $\left(\sigma_{E>1 \mathrm{MeV}}^{i}\right)^{T 3}$ and $\left(\Phi_{E>1 \mathrm{MeV}}^{i}\right)^{T 3}$ respectively the fast effective section of the dosimeter $i$, issued from the "TRIPOLI-3" calculation scheme, and the corresponding "measured" fast neutron fluence (the result of the initial dosimetric interpretation).

- $\left(\sigma_{E>1 \mathrm{MeV}}^{i}\right)^{T 4}$ and $\left(\Phi_{E>1 \mathrm{MeV}}^{i}\right)^{T 4}$ respectively the fast effective section of the dosimeter $i$, issued from the "TRIPOLI-4" calculation scheme, and the corresponding "measured" fast neutron fluence (the result of the new dosimetric interpretation).

In the TRIPOLI-4 calculation, a 3D-modeling of the whole core geometry has been performed, including the exhaustive plate-by-plate description of the OSIRIS fuel elements, in order to take into account the transport of neutrons through water layers between fuel plates (Fig. 4).

The composition of the fuel plates is fixed for each fuel element according to the burnup of the latter. The core burnup map is issued from the DAIXY code for a representative mid-cycle configuration. The radionuclide inventory as a function of burnup is previously calculated by the APOLLO-2 lattice transport code [11]. The criticality mode has been used in the TRIPOLI-4 calculation; a neutron-source distribution is only needed to initiate simulation.

Since DV50 devices ( ${ }^{\circ} 1$ and $\mathrm{n}^{\circ}$ ) were identical, one TRIPOLI- 4 calculation has been performed for both. As shown in Table 2, the fast effective cross-sections issued from the TRIPOLI-4 calculation and used in the new dosimetric interpretation of DV50 experiment are the same for DV50 $\mathrm{n}^{\circ} 1$ and DV50 $\mathrm{n}^{\circ} 2$ devices (unlike the initial dosimetric interpretation, see Table 1).

\section{Discussion}

Tables 3 presents a comparison of fast effective cross-sections issued from TRIPOLI-3 and TRIPOLI-4 calculations. Differences are less than $2 \%$ for iron, nickel and niobium dosimeters, and about $5 \%$ for 


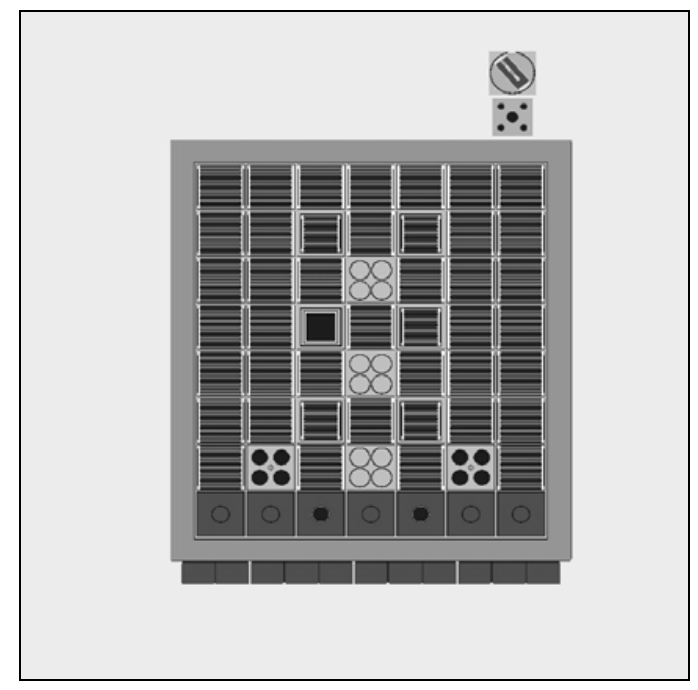

Figure 4. Geometry modeling of the OSIRIS core and the DV50 experiment with the TRIPOLI-4. The whole core geometry has been performed, including the exhaustive plate-by-plate description of the 44 fuel elements.

Table 2. Fast effective cross-sections issued from TRIPOLI-4 calculation and used in the new dosimetric interpretation (standard deviation is about $1 \%$ for $\mathrm{Fe}, \mathrm{Ni}$ and $\mathrm{Nb}$ dosimeters, and about $2 \%$ for $\mathrm{Cu}$ dosimeters). Same values are used for DV50 $\mathrm{n}^{\circ} 1$ and DV $50^{\circ} 2$ devices (unlike the initial interpretation, see Table 1).

\begin{tabular}{|c|c|c|c|c|}
\hline Dosimeter & Iron & Nickel & Niobium & Copper \\
\hline Reaction of interest & ${ }^{54} \mathrm{Fe}(\mathrm{n}, \mathrm{p}){ }^{54} \mathrm{Mn}$ & ${ }^{58} \mathrm{Ni}(\mathrm{n}, \mathrm{p}){ }^{58} \mathrm{Co}$ & ${ }^{93} \mathrm{Nb}\left(\mathrm{n}, \mathrm{n}^{\prime}\right)^{93 m} \mathrm{Nb}$ & ${ }^{63} \mathrm{Cu}(\mathrm{n}, \alpha)^{60} \mathrm{Co}$ \\
\hline New fast effective cross-section (mbarn) & 83.9 & 114.4 & 198.4 & 0.636 \\
\hline
\end{tabular}

Table 3. Comparison of fast effective cross-sections issued from TRIPOLI-3 and TRIPOLI-4 calculations.

\begin{tabular}{|c|c|c|c|c|c|}
\hline \multicolumn{2}{|c|}{ Dosimeter } & Iron & Nickel & Niobium & Copper \\
\hline Difference between fast effective & DV50 n 1 & $-0.7 \%$ & $1.9 \%$ & $1.3 \%$ & $5.1 \%$ \\
\hline cross-sections (T3-T4)/T4 & DV50 n $^{\circ} 2$ & $1.6 \%$ & $1.0 \%$ & $-0.7 \%$ & $-4.7 \%$ \\
\hline
\end{tabular}

copper dosimeters (whose threshold energy is about $6 \mathrm{MeV}$ ). Because of similarity between the crosssections of IRDF-90.V2 and IRDF-2002 libraries for the used dosimeters, the mild differences between obtained fast effective cross-sections show the consistency of neutron energy spectra calculated in the DV50 experiment with TRIPOLI-3 and TRIPOLI-4, for energies above than $1 \mathrm{MeV}$.

The final measured fast neutron fluence was the average of the four ones obtained by the 4 types of dosimeters; the difference between measured fast fluences determined by initial and new dosimetric interpretations is finally less than $3 \%$. This difference is still smaller than the measurement $1 \sigma$-uncertainty $(5 \%)$. The new interpretation does not challenge the initial one; this is an important issue.

Furthermore, similarity of fast effective cross-sections issued form TRIPOLI-3 and TRIPOLI-4 calculations does not imply necessarily similarity of calculated reaction rates and fast neutron fluxes (the fast effective cross-section is the ratio of reaction rate and fast flux).

In Table 4, we present a comparison of the nominal fast neutron flux levels $(\mathrm{E}>1 \mathrm{MeV})$ obtained at the core midplane of the DV50 experiment by measurement and by TRIPOLI-3/TRIPOLI-4 calculations. The nominal fast flux is the ratio of the fast fluence and the irradiation time expressed in equivalent full-power days (EFPD) at the OSIRIS rated power (70 MWth). The nominal fast neutron flux issued from TRIPOLI-4 calculation is in better agreement with measurements (discrepancy is about $2 \sigma$-uncertainty of measurement) than the value issued from TRIPOLI-3 calculation (discrepancy is 
Table 4. Comparison of the nominal fast neutron flux $(\mathrm{E}>1 \mathrm{MeV})$ obtained at the core midplane of the DV50 experiment by measurement and by TRIPOLI-3/TRIPOLI-4 calculations.

\begin{tabular}{|c|c|c|c|}
\hline $\begin{array}{c}\text { Nominal fast flux at the core } \\
\text { midplane }\left(\mathbf{n} . \mathrm{cm}^{-\mathbf{2}} \mathbf{s}^{\mathbf{1}}\right)\end{array}$ & $4.9 \mathrm{E} 12( \pm 5 \%)$ & $3.9 \mathrm{E} 12( \pm 3 \%)$ & $4.4 \mathrm{E} 12( \pm 1 \%)$ \\
\hline $\begin{array}{c}\text { Discrepancy between } \\
\text { calculation and measurement }\end{array}$ & - & $-20 \%$ & $-10 \%$ \\
\hline
\end{tabular}

about $4 \sigma$-uncertainty of measurement). This result would be because of a better evaluation with the new calculation scheme of the core-power map (thanks to a heterogeneous geometric model and a neutronsource issued from a critic simulation), in particular for the fuel elements located in front of the DV50 experiment an influencing directly the flux level in this latter.

So, the new calculation scheme, based on the TRIPOLI-4 code and the recent nuclear data, allows better prediction of fast flux level in vessel steel experiment irradiated in the OSIRIS reactor.

\section{Conclusion and Prospects}

In this paper, we present a new dosimetric interpretation of the DV50 experiment performed by using the Monte-Carlo code TRIPOLI-4 and more recent nuclear data (JEFF3.1.1 and IRDF-2002). Obtained results show consistency of previous and new calculated fast effective cross-sections used for dosimeter processing and hence consistency of measured fast fluences determined by initial and new dosimetric interpretations. The new interpretation does not challenge the initial one.

However, new values of calculated fast neutron fluxes are found in better agreement with measurements. So, the calculation scheme, based on the TRIPOLI-4 Monte-Carlo code and the recent nuclear data, allows better prediction of fast flux in vessel steel experiment irradiated in the OSIRIS reactor.

This work is being extended to other vessel-steel experiments carried out in the OSIRIS reactor, in order to put them at the same standard.

The author gratefully acknowledges EDF and AREVA support to TRIPOLI ${ }^{\circledR}$ (registered trademark of CEA). He also acknowledges EDF for its support to PRFLU project.

\section{References}

[1] “The OSIRIS Reactor," CEA/Saclay, http://www-centre-saclay.cea.fr/en/Labsinstitutes/

[2] Zheng S.H., Vergnaud T., Nimal J.C., "Neutron cross section probability tables in TRIPOLI-3 Monte Carlo transport Code," Nuclear science and Engineering, 126, 321-326 (1998)

[3] V. McLane, "ENDF/B-VI Summary Documentation", ENDF-201, BNL-NCS-17541, Dec., 1996.

[4] International Atomic Energy Agency, "The international Reactor Dosimetry File IRDF-90", IAEA-NDS-141, Rev. 4, March 1999

[5] “TRIPOLI-4 Monte Carlo Transport Code,” http://www .nea.fr/abs/html/nea-1716.html/

[6] A. Santamarina, D. Bernard, P. Blaise, M. Coste, A. Courcelle, T.D. Huynh, C. Jouanne, P. Leconte, O. Litaize, S. Mengelle, G. Noguère, J.M. Ruggieri, O. Sérot, J. Tommasi, C. VaglioGaudard, and J.F. Vidal, "The JEFF-3.1.1 Nuclear Data Library, JEFF Report 22, Validation Results from JEF-2.2 to JEFF-3.1.1,” NEA No. 6807, OECD/NEA Edition, 2009

[7] International Atomic Energy Agency, "International Reactor Dosimetry File 2002: IRDF-2002", Technical reports series $\mathrm{n}^{\circ} 452$, November 2006, STI/DOC/010/452, ISBN 92-0-105106-9 


\section{$15^{\text {th }}$ ISRD}

[8] H. Carcreff, A. Alberman, L. Barbot, F. Rozenblum, D. Beretz and Y. K. Lee, "Dosimetry Requirements for Pressure Vessel Steels Toughness Curve in the Ductile to Brittle Range", J. of ASTM International (JAI), Vol. 3, Issue 3, (Mar., 2006)

[9] Y.K. Lee and F. Malouch, "Analysis of OSIRIS In-Core Surveillance Dosimetry for GONDOLE Steel Irradiation Pragram by Using TRIPOLI-4 Monte Carlo Code", 13th International Symposium on Reactor Dosimetry (ISRD13), Akersloot, Netherlands, May 21-25, 2008

[10] C. Vasseur, R. Bruyere, A. Guillou and P. Quilichini, "Spécification d'un code de diffusion en géométrie XY: DAIXY”, Technical Report CEA-R-2759 (1965)

[11] R. Sanchez, I. Zmijarevic, M. Coste-Delclaux, E. Masiello, S. Santandrea, E. Martinolli, L. Villatte, N. Schwartz, N. Guler, "APOLLO2 year 2010," Nucl. Eng. Technol., Vol. 42, No. 5, (2010), pp. 474-499 